\title{
LOS PODCAST COMO MÉTODO DE APRENDIZAJE AUTÓNOMO EN EL ÁREA DE DERECHO DEL TRABAJO Y DE LA SEGURIDAD SOCIAL EN LA UNIVERSIDAD DEL PAÍS VASCO/ EUSKAL HERRIKO UNIBERTSITATEA*
}

\author{
Podcast as a Method of Autonomous Learning in Labour \\ and Social Security Law Issues at the \\ University of the Basque Country / Euskal Herriko Unibertsitatea
}

\author{
Miren Edurne López Rubia \\ TEU de Derecho del Trabajo y de la Seguridad Social \\ $\mathrm{UPV} / \mathrm{EHU}$
}

\section{ABSTRACT}

En este artículo trataré de explicar en qué ha consistido el Proyecto de Innovación Educativa "Los podcast como método de aprendizaje autónomo en la asignatura de Derecho del Trabajo y de la Seguridad Social en la Universidad del Pais Vasco / Euskal Herriko Unibertsitatea» (PIE 25, 2015-2017) —en el que hemos participado profesorado de dos Universidades ${ }^{1}$-, cómo se ha implementado, cuáles

* Este artículo se enmarca en el Proyecto de Innovación Educativa (PIE) 25-2015/2017, coordinado por la profesora Olga Fotinopoulou Basurko, dentro de la Convocatoria de Proyectos de Innovación Educativa 2015-2017 del Vicerrectorado de Estudios de Grado e Innovación de la Universidad del País Vasco/Euskal Herriko Unibertsitatea.

${ }^{1}$ De la UPV/EHU: Olga Fotinopoulou Basurko (coord.), María Aránzazu Martínez Balmaseda, Amaya Martínez de Viergol Lanzagorta, Koldo Irurzun Ugalde, Elisabet Errandonea Ulazia, Eider 
son las dificultades con las que nos hemos encontrado en ese proceso, resultados obtenidos y cuáles son las conclusiones a las que hemos llegado.

Palabras clave: podcast, Derecho del Trabajo y de la Seguridad Social, innovación educativa, aprendizaje autónomo.

Artikulu honetan "Los podcast como método de aprendizaje autónomo en la asignatura de Derecho del Trabajo y de la Seguridad Social en la Universidad del Pais Vasco / Euskal Herriko Unibertsitatea" (HBP 25, 2015-2017) izenburua duen Hezkuntza Berrikuntza Proiektuaren berri emango dut: zertan datzan proiektua, nola ezarri dugun, ezartze-prozesuan izan ditugun zailtasunak, lortutako emaitzak eta ondorioak.

Hitz gakoak: podcast, Lan eta Gizarte Segurantzaren Zuzenbidea, hezkuntza berrikuntza, ikaskuntza autonomoa.

In this article I will try to explain what has been the Educational Innovation Project "Podcast as a method of autonomous learning in the subject of Labour Law and Social Security at the University of the Basque Country / Euskal Herriko Unibertsitatea" (PIE 25, 2015-2017) —in which people of two Universities have participated-, how it has been implemented, what difficulties we have encountered in this process, the results obtained and the conclusions we have reached.

Keywords: podcast, Labour and Social Security Law, education innovation, autonomous learning.

Larrazabal Astigarraga, Miren Alazne Odriozola Landeras, Ana Isabel Zapirain Bilbao, Marta Arrue Mendizabal, María Nieves Martínez Gayoso, Garbiñe Biurrun Mancisidor y Miren Edurne López Rubia; y de la Universidad Jaume I de Castellón: Aranzazu Vicente Palacios, Sara Ruano Albertos e Inmaculada Ballester Pastor. 


\section{SUMARIO}

Sumario. 1. Introducción. 2. ¿Qué es un podcast? 3. Metodología (fases de actuación). 3.1. Seminario sobre podcast. 3.2. Elaboración y contenido de los podcast. 3.3. Implementación de los podcast. 3.3.1. Asignaturas que participaron en el Proyecto de Innovación Educativa. 3.3.2. Implementación por asignatura. 3.3.2.1. Derecho del Trabajo. 3.3.2.2. Derecho Sindical. 3.3.2.3. Lan eta Gizarte Politikak (Políticas Socio Laborales). 3.3.2.4. Prácticas Integradas I. 3.3.2.5. Ikasgelako Praktikak (Prácticas Integradas II). 3.3.2.6. Derecho del Trabajo y de la Seguridad Social II. 3.3.2.7. Seguridad y Salud Laboral. 3.4. Evaluación de la experiencia. 4. Conclusiones. 5. Bibliografía.

\section{Introducción}

La idea con la que un grupo de profesores solicitamos, primero, e implementamos, después, el Proyecto de Innovación Educativa titulado «Los podcast como como método de aprendizaje autónomo en la asignatura de Derecho del Trabajo y de la Seguridad Social en la Universidad del País Vasco / Euskal Herriko Unibertsitatea» (ref. 25) durante los cursos académicos 2015 a 2017 y del que ahora damos cuenta, era la de crear y emplear los podcast como herramienta metodológica para la enseñanza de la asignatura Derecho del Trabajo y de la Seguridad Social en la UPV/EHU. El objetivo principal perseguido era el de ir suprimiendo poco a poco las clases magistrales y las explicaciones de la asignatura y, de esa manera, poder dedicar el tiempo de clase presencial a cuestiones o a la realización de actividades o tareas que supusiesen un valor añadido para la asignatura y, consecuentemente, para el aprendizaje del alumnado de la misma. También se pretendía traducir el material al euskera, para que se pudiese cursar la asignatura en los dos idiomas oficiales en la CAV. El tercero de los objetivos que nos habíamos planteado se refería al fomento de la semipresencialidad y al aprendizaje autónomo del alumnado, en cuanto que competencia transversal en las distintas titulaciones en las que las asignaturas correspondientes al área de Derecho del Trabajo y de la Seguridad Social tienen presencia.

Sin embargo, casi desde la puesta en marcha del proyecto, nos dimos cuenta de que el planteamiento no había sido del todo correcto: en primer lugar, porque los y las participantes en el mismo impartimos diferentes asignaturas dentro del área del Derecho del Trabajo y de la Seguridad Social, por lo que el pro- 
yecto lo teníamos que haber enfocado desde el área de conocimiento y no desde una única asignatura dentro del área. En segundo lugar, porque impartimos docencia en diferentes Grados y Posgrados, de forma que las características de las asignaturas y, sobre todo, del alumnado no son las mismas. En tercer lugar, porque algunos/as de nosotros/as damos la misma asignatura en euskera y español, otros y otras solo en español, otras imparten diferentes asignaturas en diferentes lenguas..., lo cual ha dado lugar a que cada participante haya optado por implementar las actividades correspondientes al proyecto en la asignatura y en el idioma que le ha parecido mejor (no solo por contenido e idioma, también por el número de alumnos/as). Por último, y más importante, porque nos hemos dado cuenta de que facilitar por medio de podcast la materia que constituye «el grueso» de las diferentes asignaturas, lejos de facilitar el aprendizaje autónomo, lo que proporciona es mayor comodidad al alumnado y la tentación de no asistir a clase cuando las actividades previstas no sean evaluadas y se realicen «simplemente» para aumentar el conocimiento del alumnado y la autonomía en el aprendizaje.

Del mismo modo, el haber partido de situaciones tan diferentes en cuanto a las asignaturas, Grados y Posgrados en que se impartían, grupos de características diversas... nos ha hecho ver también las múltiples posibilidades que ofrecen los podcasts bien planteados y bien adaptados a cada una de las circunstancias.

Así pues, a lo largo de este artículo, intentaré explicar en qué ha consistido nuestro proyecto de innovación educativa (PIE) a través de las diferentes fases de desarrollo del mismo, cuáles son las dificultades que hemos encontrado y a qué conclusiones hemos llegado. Para todo ello, el punto de partida lo constituye el podcast.

\section{Qué es un podcast}

La palabra o término podcast deriva de la contracción de otros dos términos ingleses: «iPod (reproductor de música en formato digital comercializado por Apple Computer...) y broadcasting (radiodifusión en inglés)» (Leiva, 2007: 163)². Así, el podcast es una colección de archivos de audio con un formato concreto, mp3, al que la persona interesada puede acceder previa suscripción al mismo mediante canales RSS (Really Simply Sindication), de tal manera que esa persona lo recibe directamente en su ordenador cuando ejecuta un programa de gestión de música y podcast (Ballester et al., 2013: 103). Como se trata de un formato muy accesible y sencillo de manejar, pensamos que podíamos utilizarlo para la docencia como una herramienta más.

\footnotetext{
${ }^{2}$ Sobre el origen del podcasting léase Pińeiro (2011: 29-30).
} 
En nuestro caso, cada profesor/a ha ido colgando los podcast en eGela (plataforma on line de apoyo para la docencia en la UPV/EHU) en cada una de las asignaturas que imparte. Ha sido precisamente cada docente el/la que ha decidido el contenido y la finalidad del podcast, tal como veremos posteriormente.

\section{Metodología (fases de actuación)}

\subsection{Seminario sobre podcast}

La primera fase del proyecto consistió en la realización de un seminario sobre los podcast y su utilización en el marco de la docencia universitaria, que se celebró en la Facultad de Relaciones Laborales y Trabajo Social (Campus de Bizkaia), en mayo de 2016.

Las encargadas de dirigir el seminario fueron las profesoras Inmaculada Ballester Pastor y Arantza Vicente Palacio, procedentes de la Universidad Jaume I de Castellón, quienes ya habían realizado un proyecto similar en su propia Universidad y tenían implementado el podcast como herramienta docente desde el curso 2012-2013. Ellas nos explicaron en qué consistían sus podcast, cómo se elaboraban, qué programas informáticos utilizaban, cómo se implementaban, cuáles eran los resultados obtenidos, etc.

\subsection{Elaboración y contenido de los podcast}

No resultó nada sencillo arrancar con la elaboración de los podcast, debido, en primer lugar, a las dificultades técnicas para poder «bajar» el programa Audacity desde internet, ya que los ordenadores corporativos están sujetos a autorización. Esto es, para poder acceder a los programas gratuitos existentes on line, hay que solicitar el desbloqueo del ordenador para el programa concreto en cuestión para que, con posterioridad, alguna persona del propio Centro de Atención al Usuario (CAU) de la UPV/EHU sea quien «baje» el programa a cada ordenador. Además, una vez realizada la grabación mediante el programa Audacity y para poder guardarlo en formato $\mathrm{Mp} 3$, tuvimos que volver a solicitar autorización para conseguir el programa que hacía la conversión a Mp3. Si bien ello es así, no es menos cierto que alguna profesora del grupo de docentes asociado a este PIE pudo solucionar este inconveniente guardando el archivo en Google drive y compartiendo, después, el link correspondiente al archivo en eGela, para poder escucharlo.

Superadas las dificultades técnicas, abordamos la segunda dificultad, relacionada con el contenido del propio podcast. Parte del profesorado optó por hacer alguna práctica mediante podcast; otra parte se decantó por hacer las presentaciones de las diferentes lecciones y, por último, hubo quien, mediante podcast, grababa en audio artículos de opinión para incitar al alumnado a par- 
ticipar en el foro de debate o discusión de la asignatura. Tal y como se verá posteriormente, el hecho de no haber establecido un único tipo de contenido ha dado lugar a que la evaluación del proyecto realizado no sea, para nada, concluyente; aunque cada profesor/a ha podido valorar su aportación particular al proyecto.

\subsection{Implementación de los podcast}

\subsubsection{Asignaturas que participaron en el Proyecto de Innovación Educativa (PIE)}

La realización de tareas mediante podcast se implementó, durante el curso 2016-2017, en las siguientes asignaturas del Grado en Relaciones Laborales y Recursos Humanos:

- Derecho del Trabajo, obligatoria de 9 créditos (anual), grupos 01 y 16 de primero;

—Derecho Sindical, obligatoria de 9 créditos (anual), grupo 16 de segundo;

— Lan eta Gizarte Politikak (Políticas Socio Laborales), obligatoria de 6 créditos (cuatrimestral), grupo 31 de tercero;

— Prácticas Integradas I, obligatoria de 6 créditos (cuatrimestral), grupo 01 y 31 de tercero;

- Ikasgelako Praktikak II (Prácticas Integradas II), optativa de 6 créditos (cuatrimestral), grupo 31 de cuarto.

También se implementó en la asignatura obligatoria Derecho del Trabajo y de la Seguridad Social II, de 6 créditos, del Grado de Derecho ${ }^{3}$ y en la asignatura optativa «Seguridad y Salud Laboral» del Máster en Gestión de Recursos Humanos y el Empleo (UPV/EHU), durante el mismo curso (3 créditos).

\subsubsection{Implementación por asignatura}

\subsubsection{Derecho del Trabajo ( $\left.D^{o} T\right)$}

Derecho del Trabajo es una asignatura obligatoria de 9 créditos de carácter anual que se imparte en primer curso de Grado.

La tarea consistió en la realización de un supuesto práctico con una exposición teórica previa y se propuso a 80 alumnos/as de los dos grupos de castellano, el 01 (mañana) y el 16 (tarde). Se trataba de una tarea "semiobligatoria» en palabras de la profesora encargada de la docencia en ambos grupos, ya que, aunque la realización de la misma era voluntaria, realizarla suponía tenerla en cuenta en

\footnotetext{
3 Facultad de Derecho, Sección Bizkaia.
} 
la nota práctica. Participaron en la tarea 54 alumnos/as, es decir el $67.5 \%$ del alumnado (el $75 \%$ en el grupo 01 y el $60 \%$ en el grupo 16 ).

La profesora valoró la experiencia como positiva, porque le permitió aprender el uso de una nueva herramienta. Sin embargo, en su opinión, la utilización del podcast habría sido más útil y eficaz si lo hubiera usado solo para explicaciones teóricas.

\subsubsection{Derecho Sindical ( $\left.D^{o} S\right)$}

La asignatura Derecho Sindical es una asignatura obligatoria de 9 créditos de carácter anual y que se imparte en segundo curso de Grado. Habitualmente son dos los profesores encargados o responsables de la asignatura y el reparto docente entre ellos es por cuatrimestres.

En esta ocasión, fue el profesor que imparte docencia en el primer cuatrimestre quien planteó la resolución de un caso práctico a través de podcast. Sin embargo, lo hizo en el segundo cuatrimestre, cuya docencia está en manos de otra profesora del departamento. Ambos comparten la asignatura en eGela, por lo que todo el alumnado tuvo acceso a la práctica, que se planteó de forma voluntaria. Nadie hizo la práctica, de lo cual podrían extraerse algunas conclusiones, entre las que se pueden destacar, en primer lugar, que, al tratarse de una actividad voluntaria en la que no se ha previsto una valoración concreta de la misma, no tiene la entidad suficiente como para atraer la atención del alumnado $\mathrm{y}$, menos, cuando se trata del «otro" profesor (que ya ha evaluado su parte, aunque la han podido superar o no); y, por último, que la novedad del sistema empleado para la práctica, junto con la voluntariedad, no ha sido suficiente motivación para suscitar el interés por aprender del alumnado.

\subsubsection{Lan eta Gizarte Politikak (LGP) (Políticas Socio Laborales)}

Esta asignatura obligatoria se imparte en tercer curso de Grado, concretamente en el segundo cuatrimestre y la implementación de la tarea mediante podcast se hizo en el grupo de euskera, el 31, que cuenta, habitualmente, con un número menor de alumnado.

La tarea consistió en la realización de un ejercicio práctico. En la primera parte del podcast se proporcionó al alumnado información teórica para, a continuación, plantear una serie de cuestiones sobre dicha información. En palabras de la profesora que lo llevó a cabo, el tema era especialmente adecuado para este tipo de tarea, ya que se trataba de un tema novedoso, que no necesitaba de mucha explicación, fácilmente comprensible y que posibilitaba el planteamiento de diversos ejemplos. Se trataba, además, de una materia propia de otra asignatura del Grado, pero no impartida por falta de tiempo, por lo que daba lugar también a establecer relaciones entre distintas asignaturas del Grado; aspecto este 
que también valoramos como positivo, ya que el planteamiento de la docencia de cada asignatura tiende a hacerse como si de un compartimento estanco se tratara.

La explicación de la tarea se llevó a cabo en el aula, durante la hora de clase, y también la posterior corrección de las preguntas planteadas y las dudas surgidas, por lo que participó todo el alumnado presente en la misma (12 personas, el $66.66 \%$ del alumnado matriculado en la asignatura). Ni siquiera se planteó expresamente la obligatoriedad o no de realizar la tarea, sino que se asumió como otra más de las tareas que se hacen en clase, pero como algo novedoso en la forma de realizarla. La profesora tuvo en cuenta esta tarea en la valoración final de la asignatura.

En cuanto a la valoración del podcast como instrumento docente, el alumnado lo valoró de forma positiva, lo mismo que la profesora encargada de elaborar y dirigir la tarea, quien vio en el podcast un gran potencial para realizar diferentes actividades.

\subsubsection{Prácticas Integradas I (PI I)}

Se trata de una asignatura obligatoria, que se imparte en tercer curso de Grado durante el segundo cuatrimestre y cuyo contenido gira en torno a prácticas de Seguridad Social.

Mediante podcast publicado en eGela, se plantearon algunas cuestiones sobre maternidad. Para ello, el podcast constaba de dos partes: la primera de ellas era una introducción teórica sobre el tema y la segunda contenía preguntas sobre el contenido teórico.

La actividad se propuso a la totalidad del alumnado del grupo 01, 41 alumnos/as, y del grupo 31 (euskera), 20 alumnos/as. Además, se configuró como una tarea de ejecución obligatoria dentro del sistema de evaluación continua de la asignatura y, por tanto, computaba, como el resto de tareas, en la evaluación.

\subsubsection{Ikasgelako Praktikak II (IP II) (Prácticas Integradas II)}

Ikasgelako Praktikak II (Prácticas Integradas II) es una asignatura optativa de cuarto curso de Grado, que se imparte en el segundo cuatrimestre y cuyo contenido gira en torno a prácticas de Derecho Procesal Laboral. La dinámica habitual de la asignatura consiste en la realización de casos prácticos que se corrigen en clase. Para ello, el alumnado cuenta con todos los casos prácticos desde el comienzo de curso, ya que la profesora los "cuelga» en eGela desde el principio.

El planteamiento del caso práctico a través del podcast fue algo diferente a lo que habitualmente hacen. En esa ocasión, tenían que escuchar el podcast 
fuera del horario de clase, para hacerse una idea de las cuestiones sobre las que versaban los dos casos propuestos, pero no tenían que ir más allá.

Una vez en clase, la profesora dividió a los/as estudiantes en dos grupos diferentes, asignando a cada grupo uno de los casos propuestos. A partir de ahí, cada grupo se tenía que organizar para decidir quién sería el cliente que planteara sus preguntas/dudas/cuestiones y quiénes los que resolvieran o dieran solución a las mismas, mediante la utilización de argumentos jurídicos (normativa, jurisprudencia, doctrina).

La práctica se planteó como algo voluntario, lo cual dio lugar a que se tuviera que retrasar una semana, porque el día previsto para hacer la práctica en clase el alumnado llegó sin haber escuchado el podcast, a pesar de que la profesora les había advertido de que lo hicieran. Una semana más tarde, el alumnado presente en clase lo había escuchado y se pudo llevar a cabo. Sin embargo, las dos horas y media que duró la clase no fue suficiente para terminar la actividad, ya que, si bien entregaron por escrito cómo se habían organizado, las bases jurídicas para la resolución del caso y los documentos, la posible o posibles soluciones del caso y los comentarios del alumnado los tuvieron que dejar para otra clase.

Esta actividad, aunque voluntaria, se tuvo en cuenta en la valoración final de la asignatura.

\subsubsection{Derecho del Trabajo y de la Seguridad Social II ( $D^{o}$ Ty SS)}

En esta asignatura del Grado de Derecho, se llevaron a cabo dos tipos de actividades: por un lado, se utilizó el podcast como marco teórico para potenciar la búsqueda de información y, por otro lado, para hacer casos prácticos.

La realización de las tareas fue totalmente voluntaria y del total del alumnado matriculado, 100 personas, 70 participaron en la actividad, es decir, la totalidad del alumnado que asistía habitualmente a clase, lo que supone un $70 \%$ de participación.

La profesora valoró la experiencia de forma positiva. Son varias las razones que ofrece para llegar a dicha conclusión. Por un lado, además de usar una nueva herramienta - lo que ayuda a que el profesorado siga innovándose y adecuándose al uso de las nuevas tecnologías - , cree que, mediante el podcast, se puede ayudar a fomentar el aprendizaje autónomo del alumno/a, ya que tienen que trabajar también fuera del aula. Por otro lado, se ven obligados/as a hacer un esfuerzo extra, comprendiendo y sintetizando los datos que escuchan en el audio; esfuerzo que no se les exige cuando trabajan en formato escrito. Por último, considera que este proyecto le ha ayudado a crear unos materiales útiles e interesantes para la materia en cuestión.

Ahora bien, sin perjuicio de la valoración positiva que se extrae de su utilización, esta profesora estima que el uso del podcast es, sobre todo, recomendable, 
para la realización de prácticas o resolución de casos en los que no se requieran muchos datos; y cree que puede resultar especialmente indicado para realizar reseñas jurisprudenciales, comentarios de noticias de actualidad y profundizar en casos prácticos ya expuestos, entre otros.

\subsubsection{Seguridady Salud Laboral (SSL)}

Esta es la asignatura en la que quien suscribe estas páginas implementó el podcast como herramienta metodológica. Lo hice porque creí que, aunque se trataba de una asignatura optativa de un Máster y no de Grado, el número de alumnos/as era el adecuado (18 personas) y, sobre todo, porque me pareció que el alumnado estaba especialmente interesado y motivado ${ }^{4}$ para el seguimiento de la propia asignatura.

Utilicé el podcast para dinamizar el foro de participación a través de eGela. Durante tres semanas consecutivas, les di a conocer, a través de podcast, artículos - que podrían calificarse como científicos-, con la finalidad de que, semanalmente, comentasen su contenido, lo criticasen o aportasen sus propias opiniones sobre el tema que se trataba. La participación en el foro era obligatoria: cada alumno/a debía participar, al menos, una vez por cada uno de los temas; participación que debía hacerse por escrito. Cierto es que, en la mayoría de los casos, se limitaron a una única participación semanal, pero el contenido de cada una de las participaciones fue extensísima, es decir, superó con creces mis expectativas.

\subsection{Evaluación de la experiencia}

Además de la evaluación de la experiencia por parte del profesorado participante en el proyecto, estimamos oportuno que el alumnado que había realizado las diferentes tareas también emitiese su valoración.

$\mathrm{Al}$ profesorado le pedimos que completase un cuestionario (8 cuestiones), de forma que nos facilitase datos objetivos sobre la actividad (asignatura en la que has utilizado el podcast; número de grupos en la que lo has implementado; idioma/s del grupo; número de alumnado al que has propuesto la tarea; si la participación ha sido obligatoria o no; si ha sido una tarea voluntaria, cuántos/as han participado; y para qué tarea has utilizado el podcast: para presentar parte teórica, para realizar ejercicios prácticos, para dinamizar el foro...) y, también que valorase la experiencia.

En virtud de las valoraciones recibidas por parte del profesorado participante, la valoración es, fundamentalmente positiva.

4 También podía haber elegido la asignatura Salud Laboral y Prevención de tercero de grado, concretamente la que imparto en euskera, que es similar a la del Máster en cuanto a contenido y número de alumnos/as, sin embargo el interés y la motivación de ambos grupos difiere bastante, por no decir, mucho. 
Para poder evaluar el grado de satisfacción del alumnado, elaboramos una breve encuesta o cuestionario, que tenían que contestar vía eGela. Este fue el cuestionario (Crespo y Polo, 2009: 10-12):

Responda a cada pregunta con una valoración del 1 al 5. El 1 significa el valor mínimo, y el 5 el máximo.

1. Valore si la metodología estimula la asistencia las clases.

2. Valore si la metodología incentiva la participación del alumnado en clase.

3. La metodología empleada facilita el conocimiento y comprensión de los aspectos estructurales de la asignatura.

4. Valore si la metodología empleada fomenta el aprendizaje autónomo $\mathrm{del} / \mathrm{a}$ alumno/a.

5. Valore su participación general en el proceso de aprendizaje (autoaprendizaje y aprendizaje compartido de esta asignatura).

6. La metodología empleada permite adquirir y/o perfeccionar habilidades y competencias genéricas de utilidad en otros ámbitos sociales y/o laborales (uso de herramientas TIC's, búsqueda y captura de información, tratamiento de la información, realización de síntesis, etc.).

7. Valore si la metodología empleada es mejor que la tradicional.

8. ¿Qué valoración global realiza de la metodología utilizada?

Lo cierto es que, como se verá en los datos, no todo el alumnado participante respondió a la encuesta. De hecho, a la vista del bajo número de respuestas obtenidas, podemos adelantar que, seguramente, habría sido más prudente y, sobre todo, más eficaz, haber entregado la encuesta en papel, para cumplimentarla durante las clases.

Cuadro 1

\section{Participación alumnado}

\begin{tabular}{c|c|c|c}
\hline Asignatura & $\begin{array}{c}\text { Propuesta } \\
100 \% \text { alumnado }\end{array}$ & Participantes & Encuestas \\
\hline Do T & 80 & $54(67,5 \%)$ & $17(31,48 \%)$ \\
Do S & 71 & 0 & 0 \\
LGP & 18 & $12(66,66 \%)$ & $8(66,66 \%)$ \\
PI I & 61 & $14(77 \%)$ & $61(100 \%)$ \\
IP II & 18 & $70(70 \%)$ & $3(21.42 \%)$ \\
Do T y SS & 100 & $18(100 \%)^{*}$ & $8(35,71 \%)$ \\
SSL & 18 & $229(63,08 \%)$ & $122(53,27 \%)$ \\
\hline Total & 366 &
\end{tabular}

* Tanto la asistencia a clase como la realización de la tarea eran obligatorias.

Fuente: elaboración propia. 
A la vista de los datos, podemos afirmar que la mayor participación del alumnado depende de la obligatoriedad o de la valoración de la actividad dentro del sistema de evaluación continua. Además, para la obtención de las encuestas de la totalidad del alumnado participante, tal vez habría sido necesario incluir la entrega de la encuesta como condicionante de cara a obtener la valoración o puntuación correspondiente. Tal vez no sea lo más apropiado, pero, sin duda, nos habría dado mejor resultado.

En cuanto al grado de satisfacción del alumnado, según los datos obtenidos de las encuestas, podemos decir que, si bien en casi todas las cuestiones y asignaturas se ha superado el 2.5 (que supondría el aprobado) y que la valoración global obtenida es de 3,16, lo cierto es que las valoraciones obtenidas han sido, en general, peores de lo esperado, salvo en las asignaturas Derecho del Trabajo del Grado de Relaciones Laborales y Recursos Humanos y Derecho del Trabajo y de la Seguridad Social en el Grado de Derecho.

Sin embargo, como dato positivo, hay que destacar que las valoraciones promedio más altas coinciden con los indicadores o cuestiones 4, 5 y 6 , es decir con la valoración de la utilización del podcast como metodología que fomenta el aprendizaje autónomo $(3,28)$, con la participación general en el proceso de aprendizaje $(3,47)$ y con la valoración de la utilización del podcast como metodología que permite adquirir y/o perfeccionar habilidades y competencias genéricas de utilidad en otros ámbitos sociales y/o laborales (uso de herramientas TIC's, búsqueda y captura de información, tratamiento de la información, realización de síntesis, etc.) $(3,49)$. Todo ello nos hace pensar que el podcast es un instrumento o una herramienta totalmente válida que puede utilizarse en el proceso del aprendizaje autónomo.

Cuadro 2

Respuestas de la encuesta. Grado de satisfacción del alumnado*

\begin{tabular}{cccccccccc}
\hline & C. 1 & C. 2 & C. 3 & C. 4 & C. 5 & C. 6 & C. 7 & C. 8 & Promedio \\
\hline D $^{\circ} \mathrm{T}$ & 2,33 & 3.83 & 3,35 & 3,90 & 3,48 & 3,80 & 2,30 & 3,28 & 3,28 \\
D $^{\circ}$ S & - & - & - & - & - & - & - & - & \\
LGP & 2,37 & 3 & 2,5 & 2,87 & 2,75 & 2,87 & 3.37 & 3,00 & 2,84 \\
PI I ** & - & - & - & - & - & - & - & - & \\
IP II & 3,00 & 3,00 & 2,66 & 2,66 & 3,66 & 3,33 & 2,66 & 3,00 & 2,99 \\
DoT y SS & 3,80 & 3,48 & 3,68 & 3,88 & 3,88 & 3,84 & 4,04 & 3,64 & 3,77 \\
SSL & 2,25 & 2,25 & 2,76 & 3,12 & 3,62 & 3,62 & 2,87 & 2,87 & 2,92 \\
\hline Valoración global & 2,75 & 3,11 & 2,79 & 3,28 & 3,47 & 3,49 & 3,04 & 3.15 & 3,16 \\
\hline
\end{tabular}

* Se presenta la nota media obtenida por cada cuestión en cada una de las asignaturas y la valoración promedio en cada grupo.

** No se han podido recuperar los datos de eGela 2016-2017.

Fuente: elaboración propia. 


\section{Conclusiones}

Puedo adelantar, sin ningún lugar a dudas, que este último epígrafe bien podía haberse titulado "errores de los que aprender» en vez de "Conclusiones» 5 .

La primera conclusión a la que hemos llegado es que no se puede abordar un proyecto de innovación docente con profesorado que, si bien comparte la misma área de conocimiento, no comparte ni la misma asignatura, ni el mismo Grado o Postgrado. Ello ocurrió por querer presentar un proyecto en el que participaran muchos profesores y profesoras, dado que en su momento se consideró que un mayor número de experiencias nos aportarían más y mejores resultados y conclusiones. Nos equivocamos. Habría sido más prudente hacer un planteamiento más restringido, más limitado, que en el futuro se pudiera extender. De haberlo planteado así, se podrían haber extraído algunas conclusiones de las que poder partir, para ir ampliado el proyecto en el futuro.

Otra cuestión que no tuvimos en cuenta fue el hecho de querer implementar el proyecto tanto en grupos de castellano como en grupos de euskera, sin tener en cuenta que las características de esos grupos difieren; sobre todo en cuanto al número de alumnado. Tampoco se tuvo en cuenta que el proyecto se iba a implementar en diferentes cursos del Grado e, incluso, en un Máster oficial. Hay que reconocer que el alumnado de Grado y de Postgrado no es comparable, y tampoco lo es el alumnado de primero y el alumnado de tercero - dentro del mismo Grado-, ni en cuanto al número, ni en cuanto al interés demostrado respecto del aprendizaje. Por tanto, la segunda conclusión obtenida de nuestra experiencia es que los grupos en los que se implementa un proyecto de este tipo deben compartir un mínimo de características, de forma que luego se puedan analizar y valorar los resultados.

La tercera conclusión a la que hemos llegado está directamente relacionada con la tarea o contenido del podcast. Después de la experiencia que hemos tenido, queda claro que, si se quiere llegar a poder valorar los resultados, es necesario que la tarea sea igual o similar.

También hemos concluido que no fue un acierto dejar al arbitrio de cada docente la decisión de que la tarea a realizar fuera obligatoria o no. Aunque lo cierto es que, en este caso, la diferencia en los resultados sí ha podido valorarse, lo cierto es que la participación en la realización de la tarea ha sido mayor en aquellos grupos en los que dicha realización ha sido obligatoria o en aquellos en los que, no siendo obligatoria, la valoración de la tarea formaba parte de la nota final.

A pesar de que este planteamiento tan abierto y amplio del proyecto ha supuesto dificultades para su valoración, también hemos de admitir que ha servido

5 Me refiero a las conclusiones respecto del proyecto en sí. 
para ver las diversas y variadas utilidades del mismo. De tal forma que podemos decir que se trata de una herramienta, que bien planteada y adaptada a cada circunstancia, viene a ser un instrumento útil de aprendizaje y de trabajo autónomo para el alumnado.

\section{Bibliografía}

Ballester Pastor, Inmaculada; Vicente Palacios, Arántzazu y Ruano Albertos, Sara (2013): «Los podcast aplicados a la docencia universitaria: una experiencia en Derecho del Trabajo y de la Seguridad Social», Trabajo, núm. 29, págs. 101-117, disponible en http://rabida.uhu.es/dspace/bitstream/handle/10272/10843/Los_podcast_ aplicados.pdf?sequence $=2$ (último acceso: 12.07 .2017 ).

Crespo González, Jorge y Polo Villar, César (2009): «La evaluación de las metodologías de aprendizaje en la universidad: una experiencia desde la Universidad Complutense de Madrid", Ponencia presentada en Univest 09, II Congreso Internacional, Claves para la implicación de los estudiantes en la Universidad, Girona 12 y 13 de noviembre de 2009, disponible en http://eprints.ucm.es/11551/1/PONENCIA_232_ PRESENTADA.pdf (último acceso: 16.10.2017).

Leiva Aguilera, Javier (2007): «Podcast», Anuario ThinkEPI, págs. 163-166, disponible en: https://recyt.fecyt.es//index.php/ThinkEPI/article/view/49178/30215 (último acceso: 11.07.2017).

Piñeiro Otero, Teresa (2011): «La utilización de los podcast en la universidad española: entre la institución y la enseñanza», Hologramática. Revista Académica de la Facultad de Ciencias Sociales UNLZ, Año VII, núm. 15, V4, págs. 27-49, disponible en: http:// www.cienciared.com.ar $/ \mathrm{ra} /$ revista.php? wid $=3 \&$ articulo $=1621 \&$ tipo=A\&eid $=15 \&$ sid $=$ $136 \&$ NombreSeccion=Articulos\&Accion=Ver (último acceso: 11.07.2017).

Ruiz Miguel, José Luis: "Una ¿nueva? tecnología para el aula: el podcast», CALANDA Revista didáctico-cultural, núm. 6, 2011, págs. 75-82, disponible en: https://dialnet. unirioja.es/revista/23656/A/2011 (último acceso: 16.10.2017). http://www.mecd. gob.es/francia/publicaciones-materiales/publicaciones.html 